\title{
Einführung: Phytotherapie in der Pädiatrie und für stillende Mütter - zwischen Evidenz und praktischer Erfahrung
}

\author{
Ulrike Kastner \\ Maria Enzersdorf, Österreich
}

Welch einem brisanten Thema hat sich die Schweizerische Medizinische Gesellschaft für Phytotherapie (SMGP) gerade zu ihrer 30. Jahrestagung am 12. November 2015 in Baden gewidmet!

Die Behandlung von Kindern, Jugendlichen und Stillenden ist und bleibt eine Herausforderung für alle beteiligten Disziplinen, Ärzte sowie Apotheker, hat man es doch mit einer Patientengruppe zu tun, die aufgrund ihres Alters oder aufgrund der aussergewöhnlichen Lebensumstände als besonders vulnerabel und schützenswert gilt.

Pflanzliche Arzneimittel haben den Ruf, dass sie aufgrund des «natürlichen Ursprungs» nebenwirkungsarme und milde Arzneimittel sind. Doch Naturstoffgemische, wie wir sie in Phytotherapeutika oder auch traditionellen Rezepturen finden, sind nun einmal nicht immer frei von unerwünschten Wirkungen.

Dieser kritischen Betrachtung stellten sich im Rahmen der 30. Jahrestagung Experten aus unterschiedlichen Disziplinen, um die derzeitige Lage der Behandlungsmöglichkeiten in Theorie und Praxis zu beleuchten.

Obgleich in der EG-Verordnung Nr. 1901/2006 über Kinderarzneimittel die Zulassungsanforderungen für die Altersgruppe der unter 12- bzw. 18-Jährigen spezifiziert wurden, in der Hoffnung eine bessere Studienlage zu erhalten, ist es gerade für pflanzliche Arzneimittel extrem schwierig geworden, diese Anforderungen zu erfüllen. Abgesehen von ethischen Aspekten sind Studien an Kindern oder stillenden Müttern schwer realisierbar, und es mangelt an finanziellen Mitteln und Studienzentren, die bereit sind, auch Arzneimittel bei milden bis moderaten Krankheitsbildern zu prüfen. So bleibt vieles immer noch in der Verantwortung des Verschreibenden, der aufgrund seiner Erfahrung mit Phytotherapie und seines Wissens um die Inhaltsstoffe der unterschiedlichen Drogen im Sinne des Patienten handeln muss. "Off-label use» - und das sind teilweise bis zu 50\% der Verschreibungen - ist eine Realität in der pädiatrischen Praxis geworden und wird nicht mehr still, nein, zunehmend auch fordernd hingenommen.

\section{KARGER}

Fax +497614520714

Information@Karger.com

www.karger.com
In der Schweiz ist - anders als in vielen europäischen Ländern - eine grosse Anzahl pflanzlicher Arzneimittel mit offiziell von Swissmedic genehmigten Patienteninformationen und Dosisanleitungen versehen. Sie sind in sehr übersichtlicher Form in einer Tabelle von Vizepräsidentin Frau Dr. Beatrix Falch zusammengestellt worden und über die Website der SMGP (www.smgp.ch) abrufbar. Bezüglich der pflanzlichen Arzneimittel in der Stillzeit sei auch auf die umfassende Datenbank «Herba pro Matre» und die unabhängige Arbeitsgruppe «Phyto-Gyni-Netzwerk Herbadonna», ebenfalls unter der Leitung von Frau Dr. Falch, hingewiesen.

Der ganzheitliche Ansatz in der Therapie von Kindern und Jugendlichen setzt voraus, dass man die Pathogenese häufiger Krankheitsbilder, wie Atemwegsinfektionen, Hauterkrankungen sowie Erkrankungen des Magen-DarmTrakts und des Nervensystems, verstehen lernt. Nur durch das Verständnis der Auslöser, wie z.B. bei Allergien, Asthma bronchiale, Neurodermitis usw., kann Phytotherapie zielgerichtet und mit naturwissenschaftlichem Hintergrund eingesetzt werden. Natürlich basiert vieles immer noch auf traditionellem Wissen, und dies wird auch auf absehbare Zeit so bleiben, aber das Bestreben um mehr Aufklärung der Struktur-Wirkungsbeziehungen und somit auch um Therapiesicherheit für den Patienten ist spürbar und zeigt immer wieder neue Erkenntnisse bezüglich der Wirksamkeit der komplexen Pflanzeninhaltsstoffe. So manch traditioneller Ansatz, wie z.B. der Einsatz der Karottensuppe nach Moro in der Behandlung von kindlichen Durchfallerkrankungen, kann heute durch entsprechend phytochemische und pharmakologische Erkenntnisse erklärt werden und bestärkt den Behandelnden in seiner Therapieempfehlung.

In diesem Sinne: Tauchen Sie ein in die Beiträge zum Thema «Phytotherapie in der Pädiatrie und für stillende Mütter». Nur Wissens- und Erfahrungsaustausch stellen sicher, dass Kindern und Jugendlichen sowie stillenden Müttern auch weiterhin pflanzliche Arzneimittel mit hoher Qualität und Wirkungsanspruch zur Verfügung stehen und das traditionelle Wissen nicht verloren geht! 